\title{
IMAGE FILLING-IN IN A DECOMPOSITION SPACE
}

\author{
Marcelo Bertalmio, Luminita Vese, Guillermo Sapiro, Stanley Osher \\ University of Pompeu-Fabra, University of Minnesota, UCLA \\ guille@ece.umn.edu
}

\begin{abstract}
An algorithm for the simultaneous filling-in of texture and structure in regions of missing image information is presented in this paper. The basic idea is to first decompose the image into the sum of two functions with different basic characteristics, and then reconstruct each one of these functions separately with structure and texture filling-in algorithms. The first function used in the decomposition is of bounded variation, representing the underlying image structure, while the second function captures the texture and possible noise. The region of missing information in the bounded variation image is reconstructed using image inpainting algorithms, while the same region in the texture image is filledin with texture synthesis techniques. The original image is then reconstructed adding back these two sub-images. The novel contribution of this paper is then in the combination of these three previously developed components, image decomposition with inpainting and texture synthesis, which permits the simultaneous use of filling-in algorithms that are suited for different image characteristics. The novelty in the approach is to perform filling-in in a domain different from the original given image space. Examples on real images show the advantages of this proposed approach.
\end{abstract}

\section{INTRODUCTION AND ALGORITHM OVERVIEW}

The filling-in of missing information is a very important topic in image processing, with applications including image coding and wireless image transmission (e.g., recovering lost blocks), special effects (e.g., removal of objects), and image restoration (e.g., scratch removal). The basic idea behind the algorithms that have been proposed in the literature is to fill-in these regions with available information from their surroundings. This information can be automatically detected as in $[3,7]$, or hinted by the user as in more classical texture filling techniques $[6,8,14]$.

The algorithms reported in the literature best perform for pure texture, [7, 8, 14], or pure structure, $[1,2,3,5]$ (see also early work in [12], which shows the use of the Burt-Adelson pyramid for the reconstruction of smooth regions). This means that for ordinary images such as the one in Figure 1, different techniques work better for different parts. In [13], it was shown how to automatically switch between the pure texture and pure structure filling-in process. This is done by analyzing the area surrounding the region to be filled-in (inspired by [9]), and selecting either a texture synthesis or a structure inpainting technique. Since most image areas are not pure texture or pure structure, this approach provides just a first attempt in the direction of simultaneous texture and structure filling-in (attempt which was found sufficient for the particular application of transmission and coding presented in the paper). It is the goal of this paper to advance in this direction and propose a new technique that will perform both texture synthesis and structure inpainting in all regions to be filled-in.

The basic idea of our algorithm is presented in Figure 2, which shows a real result from our approach. The original image (first row) is first decomposed into the sum of two images, one capturing the basic image structure and one capturing the texture (and random noise), second row. This follows the recent work by Vese and Osher reported in [15] (which is inspired by [11]). The first image is inpainted following the work by Bertalmio-Sapiro-CasellesBallester described in [3], while the second one is filled-in with a texture synthesis algorithm following the work by Efros and Leung in [7], third row. The two reconstructed images are then added back together to obtain the reconstruction of the original data, fourth row. In other words, the general idea is to perform structure inpainting and texture synthesis not on the original image, but on a set of images with very different characteristics that are obtained from decomposing the given data. The decomposition is such that it produces images suited for these two reconstruction algorithms. This approach outperforms both image inpainting and texture synthesis when applied separately.

The proposed algorithm has then three main building blocks: Image decomposition, image (structure) inpainting, and texture synthesis. The basic characteristics of these components are:

1. The image decomposition is not a simple low-pass+highpass decomposition, since both images contain high frequencies, one in the form of edges and the other in the form of oscillations.

2. The image inpainting part performs very good for non-textured images, as the BV one obtained from the decomposition. This image inpainting is based on high order partial differential equations that propagate information surrounding the hole in the direction of the isophotes.

3. The texture synthesis algorithms performs very good for textures, as the oscillatory part obtained from the decomposition. Moreover, this can be enhanced with the BV part [4]. This particular algorithm works by filling-in pixels inside the hole one at a time, based on matching their surrounding neighborhood with the available image information.

The particular selections for each one of these parts, which have been shown to produce state-of-the-art results in each one of their particular applications, outperform previously available techniques when combined as proposed in this paper. In the concluding remarks section we discuss the possible use of other approaches to address each one of these building blocks in order to further improve on the results here reported.

Full details of the algorithm, extensions to color images, and comparison with pure image inpainting and pure image synthesis 
can be found in [4] and at mountains.ece.umn.edu/ guille/inpainting.htm[7] A. A. Efros and T. K. Leung, "Texture synthesis by nonparametric sampling," IEEE International Conference on Computer Vision, Corfu, Greece, pp. 1033-1038, Sept. 1999.

\section{CONCLUSIONS AND FUTURE DIRECTIONS}

In this paper we have shown the combination of image decomposition with image inpainting and texture synthesis. The basic idea is to first decompose the image into the sum of two functions, one that can be efficiently reconstructed via inpainting and one that can be efficiently reconstructed via texture synthesis. This permits the simultaneous use of these reconstruction techniques in the image domain they were designed for. In contrast with previous approaches, both image inpainting and texture synthesis are applied to the region of missing information, only that they are applied not to the original image representation but to the images obtained from the decomposition. The obtained results outperform those obtained when only one of the reconstruction algorithms is applied to each image region.

Further experiments are to be carried out to obtain the best combination of image decomposition, image inpainting, and texture synthesis. Since a number of algorithms exist for each one of these three key components, the combination that provides the best visual results is an interesting experimental and theoretical research topic.

\section{Acknowledgments}

This work was partially supported by the Office of Naval Research, the National Science Foundation, the National Institute of Health, the Office of Naval Research Young Investigator Award, the Presidential Early Career Awards for Scientists and Engineers (PECASE), a National Science Foundation CAREER Award, and by the Programa Ramon y Cajal (Ministerio de Ciencia y Tecnologia, Spain). MB and GS thank Prof. Vicent Caselles, with whom all this work on image inpainting started. He is a constant source of intellectual and personal inspiration.

\section{REFERENCES}

[1] C. Ballester, M. Bertalmio, V. Caselles, G. Sapiro, and J. Verdera, "Filling-in by joint interpolation of vector fields and grey levels," IEEE Trans. Image Processing 10, pp. 12001211, August 2001.

[2] M. Bertalmio, A. L. Bertozzi, and G. Sapiro, "Navier-Stokes, fluid dynamics, and image and video inpainting," Proc. IEEE Computer Vision and Pattern Recognition (CVPR), Hawaii, December 2001.

[3] M. Bertalmio, G. Sapiro, V. Caselles, and C. Ballester, "Image inpainting," Computer Graphics (SIGGRAPH 2000), pp. 417424, July 2000.

[4] M. Bertalmio, L. Vese, G. Sapiro, and S. Osher, "Simultaneous structure and texture image inpainting," IEEE Trans. Image Processing, to appear.

[5] T. Chan and J. Shen, "Local inpainting models and TV inpainting," SIAM J. Appl. Math. 62:3, pp. 1019-1043, 2001.

[6] J. S. De Bonet, "Multiresolution sampling procedure for analysis and synthesis of texture images," Proceedings of ACM SIGGRAPH, July 1997.
[8] D. Heeger and J. Bergen, "Pyramid based texture analysis/synthesis," Computer Graphics (SIGGRAPH 1995), pp. 229-238, July 1995.

[9] K. Karu, A. K. Jain, and R. M. Bolle, "Is there any texture in the image?," Pattern Recognition 29:9, pp. 1437-1446, 1996.

[10] S. Masnou and J. Morel, "Level-lines based disocclusion," IEEE Int. Conf. Image Processing, October 1998.

[11] Y. Meyer, Oscillating Patterns in Image Processing and Nonlinear Evolution Equations, AMS University Lecture Series 22, 2002.

[12] J. M. Ogden, E. H. Adelson, J. R. Bergen, and P. J. Burt, "Pyramid-based computer graphics," RCA Engineer 30(5), pp. 4-15, 1985.

[13] S. Rane, G. Sapiro, and M. Bertalmio, "Structure and texture filling-in of missing image blocks in wireless transmission and compression applications," IEEE Trans. Image Processing, to appear.

[14] E. Simoncelli and J. Portilla, "Texture characterization via joint statistics of wavelet coefficient magnitudes," Proc. 5th IEEE Int'l Conf. on Image Processing, 1998.

[15] L. Vese and S. Osher, "Modeling textures with total variation minimization and oscillating patterns in image processing," Journal of Scientific Computing, to appear.

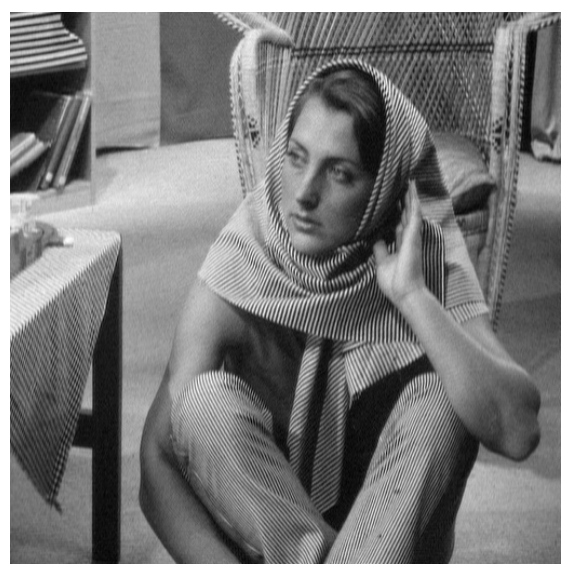

Fig. 1. Example of image with both texture and structure. 


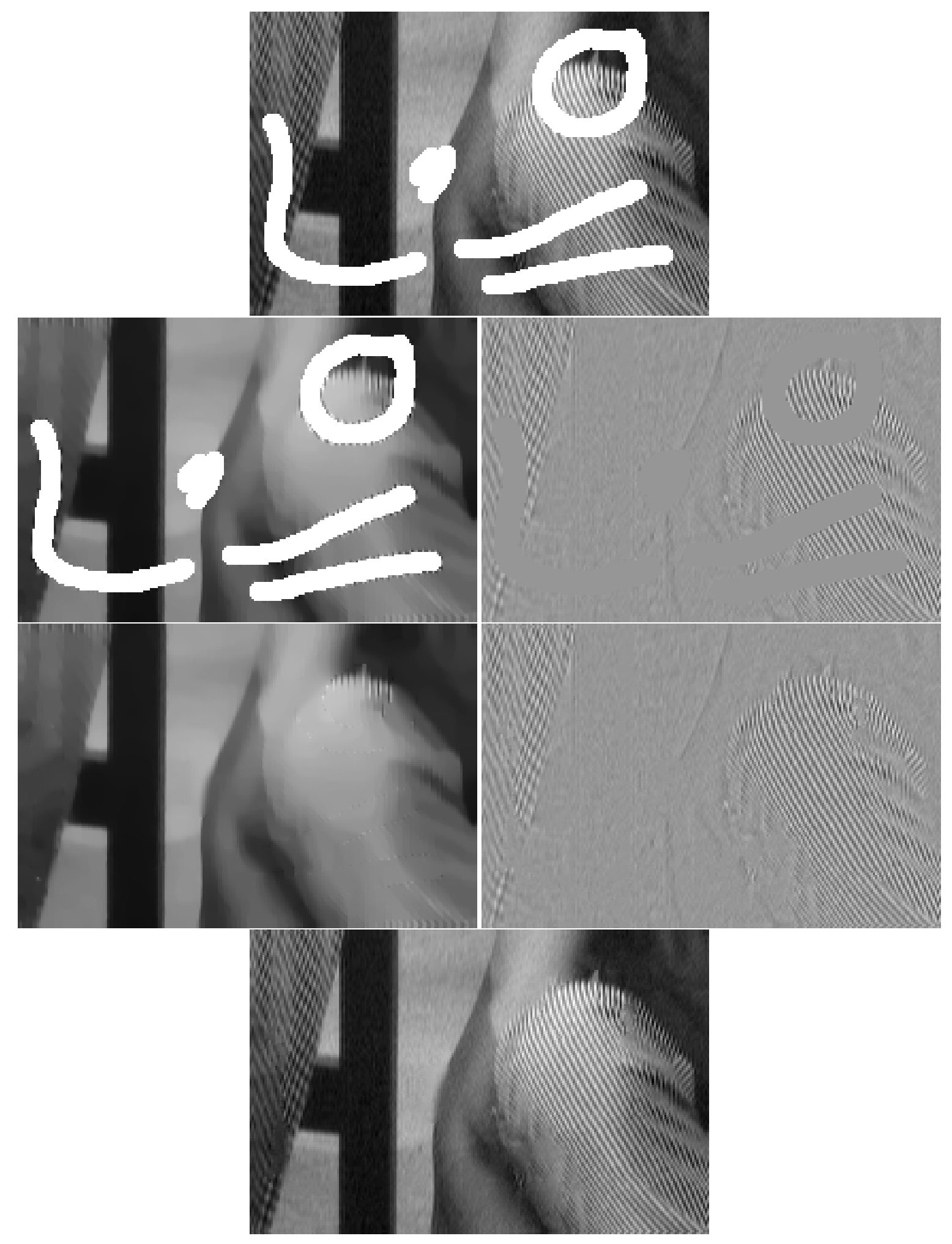

Fig. 2. Basic algorithm proposed in this paper. The original image in the first row (a section of Figure 1) is decomposed into a structure image and a texture image, [15], second row. Note how the image on the left mainly contains the underlying image structure while the image on the right mainly contains the texture. These two images are reconstructed via inpainting, [3], and texture synthesis, [7], respectively, third row. The image on the left managed to reconstruct the structure (see for example the chair vertical leg), while the image on the right managed to reconstruct the basic texture. The resulting two images are added to obtain the reconstructed result, last row, where both structure and texture are recovered. 